\title{
Modular Equations and Distortion Functions
}

\author{
G. D. Anderson, S.-L. Qiu, and M. Vuorinen
}

\begin{abstract}
Modular equations occur in number theory, but it is less known that such equations also occur in the study of deformation properties of quasiconformal mappings. The authors study two important plane quasiconformal distortion functions, obtaining monotonicity and convexity properties, and finding sharp bounds for them. Applications are provided that relate to the quasiconformal Schwarz Lemma and to Schottky's Theorem. These results also yield new bounds for singular values of complete elliptic integrals.
\end{abstract}

Keywords and Phrases: Grötzsch ring, elliptic integral, distortion function, quasiconformal, quasiregular, quasisymmetric, quasi-Möbius, Schottky's Theorem, monotonicity, convexity.

2000 Mathematics Subject Classification: Primary 30C62; Secondary 33E05.

\section{Introduction.}

For $r \in(0,1)$, let $\mu(r)$ denote the modulus of the plane Grötzsch ring $B \backslash[0, r]$, where $B$ is the unit disk. Then [20, p. 60]

$$
\mu(r)=\frac{\pi}{2} \frac{\mathcal{K}^{\prime}(r)}{\mathcal{K}(r)}
$$

where

$$
\mathcal{K}=\mathcal{K}(r) \equiv \int_{0}^{\pi / 2}\left(1-r^{2} \sin ^{2} t\right)^{-1 / 2} d t, \mathcal{K}^{\prime}=\mathcal{K}^{\prime}(r) \equiv \mathcal{K}\left(r^{\prime}\right)
$$

$r^{\prime}=\sqrt{1-r^{2}}, 0<r<1$, are complete elliptic integrals of the first kind [11], [12], [13], [40]. In the sequel, we shall also need the complete elliptic integrals of the second kind

$$
\mathcal{E}=\varepsilon(r) \equiv \int_{0}^{\pi / 2}\left(1-r^{2} \sin ^{2} t\right)^{1 / 2} d t, \quad \varepsilon^{\prime}=\varepsilon^{\prime}(r) \equiv \varepsilon\left(r^{\prime}\right),
$$

for $r \in[0,1], r^{\prime}=\sqrt{1-r^{2}}$.

The function $\mu(r)$ and the distortion functions

$$
\left\{\begin{array}{l}
\varphi_{K}(r) \equiv \mu^{-1}(\mu(r) / K) \\
\varphi_{K}(0)=1-\varphi_{K}(1)=0
\end{array}\right.
$$


for $r \in(0,1)$ and $K \in(0, \infty)$, and

$$
\lambda(K) \equiv\left[\frac{\varphi_{K}(1 / \sqrt{2})}{\varphi_{1 / K}(1 / \sqrt{2})}\right]^{2}=\frac{1-u^{2}}{u^{2}}, u=\varphi_{1 / K}(1 / \sqrt{2}),
$$

$K \in(0, \infty)$, play an important role in the study of deformation properties of quasiconformal maps [2], [7], [16], [18], [20], [22], [26], [38]. Note that here the identity (1.22) for $\varphi_{K}$ was used. These functions have also found applications in some other mathematical fields such as number theory [6], [9], [10], [11], [24], [37], [39].

If $R$ is a rectangle with sides parallel to the $x$ - and $y$-axes of length $a$ and $b$, respectively, the modulus of $R$ is defined as $M(R)=b / a$. If $Q$ is a (topological) quadrilateral, its modulus is defined as $M(Q)=M(R)$, where the rectangle $R$ is a conformal image of $Q$ and has sides parallel to the coordinate axes. The modulus of a quadrilateral is a conformal invariant.

Now let $f$ be a diffeomorphism of a domain $G$ in the $z$-plane onto a domain $G^{\prime}$ in the $w$-plane. The maximal dilatation at a point $z \in G$ is defined as

$$
K(z)=\inf _{G_{z}} \sup _{\bar{Q} \subset G_{z}} \frac{M\left(Q^{\prime}\right)}{M(Q)}
$$

where $G_{z}$ denotes any subdomain containing $z, Q$ any quadrilateral with $\bar{Q} \subset G_{z}$, and $Q^{\prime}$ the image of $Q$ under $f$. If $K(z) \leq K, 1 \leq K<\infty$, for each $z \in G$, then $f$ is called a (differentiable) $K$-quasiconformal mapping. The definition of quasiconformality can be extended to the case of a homeomorphism $f$ that may fail to be differentiable at some points. Next, for each $z \in G$ with $z \neq \infty \neq f(z)$, let

$$
H(z)=\limsup _{r \rightarrow 0} \frac{\max _{\varphi}\left|f\left(z+r e^{i \varphi}\right)-f(z)\right|}{\min _{\varphi}\left|f\left(z+r e^{i \varphi}\right)-f(z)\right|} .
$$

If $z$ or $f(z)$ is infinite, the definition of $H(z)$ can be modified in an obvious way by means of inversion. If $f$ is continuously differentiable and has a non-zero Jacobian at $z$, then $H(z)=$ $K(z)$ (see [21]). In [21], Lehto, Virtanen, and Väisälä proved that always $H(z) \leq \lambda(K(z))$, where $\lambda(K)$ is given by (1.5), and this is the best possible result of this type. Moreover, the boundary values of a $K$-quasiconformal automorphism $f$ of the upper half plane satisfy the inequality

$$
\frac{1}{\lambda(K)} \leq \frac{f(x+t)-f(x)}{f(x)-f(x-t)} \leq \lambda(K)
$$

for all real $x$ and $t, t \neq 0$, see [20, p. 81]. Accordingly, several results in distortion theory of quasiconformal mappings depend on $\lambda(K)$. One of the key areas of $K$-quasiconformal theory is the study of what happens when $K \rightarrow 1$. Quantitative study of this subject requires explicit estimates for special functions, such as we are providing in this paper.

A modular equation of degree $\mathrm{p}$ is defined as

$$
\frac{\mathcal{K}^{\prime}(s)}{\mathcal{K}(s)}=p \frac{\mathcal{K}^{\prime}(r)}{\mathcal{K}(r)},
$$

with $s, r \in(0,1)$. Its solution is $s=\varphi_{1 / p}(r)$. Ramanujan introduced the convenient notation

$$
\alpha=r^{2}, \beta=s^{2}
$$


for use in connection with modular equations. With this notation, a classical third-degree modular equation $[11$, p. 105, (4.1.16)] reads as

$$
(\alpha \beta)^{1 / 4}+((1-\alpha)(1-\beta))^{1 / 4}=1,
$$

with $\alpha=r^{2}, \beta=\varphi_{1 / 3}(r)^{2}$. Ramanujan found numerous algebraic identities satisfied by $\alpha$ and $\beta$ for several prime numbers $p$, see [9]. Ramanujan also formulated, in unpublished notes without proofs, many generalized modular equations. Proofs were published in the landmark paper [10] by Berndt, Bhargava, and Garvin.

For each positive integer $p$, there exists a unique number $k_{p} \in(0,1)$ such that

$$
\mu\left(k_{p}\right)=\frac{\pi \mathcal{K}^{\prime}\left(k_{p}\right)}{2 \mathcal{K}\left(k_{p}\right)}=\frac{\pi}{2} \sqrt{p}
$$

The number $k_{p}$ is algebraic and is called the $p$ th singular value of $\mathcal{K}(r)$ [11, pp. 139, 296]. Since $\mu(1 / \sqrt{2})=\pi / 2$ we see that

$$
k_{p}=\mu^{-1}(\sqrt{p} \mu(1 / \sqrt{2}))=\varphi_{1 / \sqrt{p}}(1 / \sqrt{2}) .
$$

The many well-known facts about $\varphi_{K}$ yield information about $k_{p}$. For instance, the infinite product expansion in [7, Theorem $5.48(3)]$ and the inequalities in $[7$, Theorem $10.9(1)]$, [29], or [30] imply such results. Note that it follows from (1.5) that $k_{p}=1 / \sqrt{1+\lambda(\sqrt{p})}$. Many singular values have been found explicitly, because they have a significant role in number theory. The algebraic numbers $k_{p}, p=1, \ldots, 9$, are given in [11, p. 139]. The values of $\mathcal{K}\left(k_{p}\right), p=1, \ldots, 16$, appear in [11, p. 298]; from these one also obtains $\mathcal{K}^{\prime}\left(k_{p}\right), p=1, \ldots, 16$.

In 1968, S. Agard [1] introduced the following generalization of $\lambda(K)$ :

$$
\eta_{K}(t)=\left[\frac{\varphi_{K}(r)}{\varphi_{1 / K}\left(r^{\prime}\right)}\right]^{2}, \quad r=\sqrt{\frac{t}{1+t}}, \quad r^{\prime}=\frac{1}{\sqrt{1+t}},
$$

for $K, t \in(0, \infty)$. He showed that

$$
\eta_{K}(t)=\sup _{f \in \mathcal{F}}\{|f(z)|:|z|=t\}
$$

where $\mathcal{F}=\left\{f: f\right.$ is a $K$-quasiconformal automorphism of the plane $\mathbb{R}^{2}$ with $f(0)=$ $f(1)-1=0\}, K \geq 1$. Clearly $\eta_{K}(1)=\lambda(K)$. The function $\eta_{K}(t)$, called the $\eta$-distortion function, has many important applications in the study of quasiconformal, quasiregular, quasisymmetric, and quasi-Möbius mappings, and Möbius-invariant metrics [1], [6], [20], [32], [33], [34], [35], [36]. Recently, G. Martin proved [23, Theorem 1.1] that

$$
\eta_{K}(t)=\sup \{|f(z)|: f \in \mathcal{A}(t),|z|=(K-1) /(K+1)\},
$$

where $t>0$ and

$$
\mathcal{A}(t)=\left\{f: B \rightarrow \mathbb{R}^{2} \backslash\{0,1\} \mid f \text { is analytic with }|f(0)|=t\right\} .
$$


Hence, in the notation used in Schottky's Theorem [15, p. 702],

$$
|f(z)| \leq \eta_{K}(a) \equiv \Psi(a,|z|), K=(1+|z|) /(1-|z|),
$$

for $f \in \mathcal{A}(a), a>0$, and $z \in B$. Upper bounds have been obtained for the function $\Psi(a, r)$ by W. K. Hayman [14], J. Jenkins [19], J. Hempel [17], and S. Zhang [43], while Zhang's estimates were recently improved in [27].

In the past few years, many properties have been derived for the special functions $\varphi_{K}(r), \lambda(K)$, and $\eta_{K}(t)$ [3], [4], [5], [6], [7], [23], [25], [27], [29], [30], [31], [32], [37], [39], [41], [42]. However, the above-mentioned applications motivate us to study these functions further. For the applications, for example, we require better estimates and some other properties for $\eta_{K}(t)$.

Since $\eta_{K}(t)$ is a generalization of $\lambda(K)$, it is natural to ask how to extend the known results for $\lambda(K)$ to $\eta_{K}(t)$. For example, $\lambda(K)$ has the following asymptotic expansion [7, Exercise 10.41 (6)] (cf. [20, (6.10), p. 82]):

$$
\lambda(K)=\frac{1}{16} e^{\pi K}-\frac{1}{2}+\delta(K), \text { where } 1<e^{\pi K} \delta(K)<2,
$$

for $K>1$. In Theorem 4.7 and Corollary 4.16 below we provide an analogue of (1.10) for the function $\eta_{K}(t)$.

In the present paper, the authors study the monotonicity and convexity properties of certain combinations of the functions $\lambda(K)$ and $\eta_{K}(t)$, from which sharp bounds for these functions follow. Note that, by (1.9), our sharp bounds for $\eta_{K}(t)$ also give sharp estimates for the Schottky upper bound $\Psi(t, r)$. Our main results include the following Theorem 1.11, which leads to an approximation of $\lambda(K)$ by a finite Taylor series, and Theorem 1.14, in which we extend to $\eta_{K}(t)$ the properties of $\lambda(K)$ proved in [32, Lemma 3.54]. Recalling that $k_{p}=1 / \sqrt{1+\lambda(\sqrt{p})}, p=1,2,3, \ldots$, we see that the bounds for $\lambda(K)$ in the next theorem also give bounds for the singular values $k_{p}$. As far as we know, the resulting bounds for singular values are new.

1.11. Theorem. Let $a=(4 / \pi) \mathcal{K}(1 / \sqrt{2})^{2}=4.3768 \ldots$ and $c=a\left[4(a-1)^{2}-a^{2}\right] / 16=$ $7.2372 \ldots$ Then the function

$$
f(K) \equiv(K-1)^{-3}\left[\lambda(K)-1-a(K-1)-\frac{1}{2} a(a-1)(K-1)^{2}\right]
$$

is strictly increasing from $(1, \infty)$ onto $(c, \infty)$. In particular, for $K>1$,

$$
\lambda(K)>1+a(K-1)+\frac{1}{2} a(a-1)(K-1)^{2}+c(K-1)^{3}
$$

and, for $K \in(1,2)$,

$$
\left\{\begin{aligned}
1+a(K-1)+\frac{1}{2} a(a-1)(K-1)^{2}+c(K-1)^{3}<\lambda(K) \\
<1+a(K-1)+\frac{1}{2} a(a-1)(K-1)^{2}+c_{1}(K-1)^{3}
\end{aligned}\right.
$$


where $c_{1}=\lambda(2)-1-a-\frac{1}{2} a(a-1)=4 \sqrt{2}(\sqrt{2}+1)^{2}-1-a-\frac{1}{2} a(a-1)=20.2035 \ldots$

1.14. Theorem. For $t \in(0, \infty)$, let $r=\sqrt{t /(1+t)}$.

(1) The function

$$
f(K) \equiv \eta_{K}(t) e^{-2 K \mu\left(r^{\prime}\right)}
$$

is strictly increasing from $[1, \infty)$ onto $\left[t e^{-2 \mu\left(r^{\prime}\right)}, 1 / 16\right)$.

(2) The function

$$
g(K) \equiv \frac{1}{\eta_{K}(t)} \frac{\partial \eta_{K}(t)}{\partial K}
$$

is strictly decreasing from $(1, \infty)$ onto $\left(2 \mu\left(r^{\prime}\right),(4 / \pi) \mathcal{K}(r) \mathcal{K}^{\prime}(r)\right)$. In particular, for all $t \in(0, \infty)$ and $K \in(1, \infty)$,

$$
t e^{2(K-1) \mu\left(r^{\prime}\right)}<\eta_{K}(t)<t e^{4(K-1) \mathcal{K}(r) \mathcal{K}^{\prime}(r) / \pi} .
$$

(3) The function

$$
h(K) \equiv e^{-2 K \mu\left(r^{\prime}\right)} \frac{\partial \eta_{K}(t)}{\partial K}
$$

is strictly increasing from $(1, \infty)$ onto $\left((4 / \pi) t \mathcal{K}(r) \mathcal{K}^{\prime}(r) e^{-2 \mu\left(r^{\prime}\right)}, \mu\left(r^{\prime}\right) / 8\right)$. In particular, for all $t \in(0, \infty)$ and $K \in(1, \infty)$,

$$
t+t\left(\frac{2}{\pi} \mathcal{K}^{\prime}(r)\right)^{2}\left[e^{2(K-1) \mu\left(r^{\prime}\right)}-1\right]<\eta_{K}(t)<t+\frac{1}{16} e^{2 \mu\left(r^{\prime}\right)}\left[e^{2(K-1) \mu\left(r^{\prime}\right)}-1\right]
$$

and

$$
1+c\left(e^{\pi K}-e^{\pi}\right)<\lambda(K)<1+\frac{1}{16}\left(e^{\pi K}-e^{\pi}\right),
$$

where $c=\left(\frac{2}{\pi} \mathcal{K}(1 / \sqrt{2})\right)^{2} e^{-\pi}=0.0602 \ldots$

Throughout this paper, we let $r^{\prime}=\sqrt{1-r^{2}}$ for $r \in[0,1]$.

We shall frequently employ the following well-known formulas [7, Appendix E], [11], [16], [20], [40], for $0<r<1, \quad 0<K<\infty$ :

$$
\begin{gathered}
\frac{d \mathcal{K}}{d r}=\frac{\mathcal{E}-r^{\prime 2} \mathcal{K}}{r r^{\prime 2}}, \quad \frac{d \mathcal{E}}{d r}=\frac{\mathcal{E}-\mathcal{K}}{r}, \\
\mathcal{K} \mathcal{E}^{\prime}+\mathcal{K}^{\prime} \mathcal{E}-\mathcal{K} \mathcal{K}^{\prime}=\frac{\pi}{2}, \\
\frac{d \mu(r)}{d r}=-\frac{\pi^{2}}{4 r r^{\prime 2} \mathcal{K}(r)^{2}}, \\
\left\{\begin{array}{l}
\frac{\partial s}{\partial r}=\frac{s}{K r}\left(\frac{s^{\prime} \mathcal{K}(s)}{r^{\prime} \mathcal{K}(r)}\right)^{2}=\frac{s s^{\prime 2} \mathcal{K}(s) \mathcal{K}^{\prime}(s)}{r r^{\prime 2} \mathcal{K}(r) \mathcal{K}^{\prime}(r)}, \\
\frac{\partial s}{\partial K}=\frac{2}{\pi K} s s^{\prime 2} \mathcal{K}(s) \mathcal{K}^{\prime}(s),
\end{array}\right.
\end{gathered}
$$

where $s=\varphi_{K}(r)$,

$$
\varphi_{K}(r)^{2}+\varphi_{1 / K}\left(r^{\prime}\right)^{2}=1, \quad \varphi_{2}(r)=\frac{2 \sqrt{r}}{1+r} .
$$


We denote

$$
m(r)=\frac{2}{\pi} r^{\prime 2} \mathcal{K}(r) \mathcal{K}^{\prime}(r),
$$

for $r \in(0,1)$. Then, by differentiation and (1.19), we have

$$
\frac{d m(r)}{d r}=\frac{1}{r}-\frac{4}{\pi r} \varepsilon^{\prime}(r) \mathcal{K}(r) .
$$

\section{Preliminary results.}

In this section we prove two lemmas that will be needed for the proofs of the main theorems in Sections 3 and 4.

2.1. Lemma. (1) The function $f(r) \equiv r r^{\prime} \mathcal{K}(r) \mathcal{K}\left(r^{\prime}\right)$ is strictly increasing on $(0,1 / \sqrt{2}]$, and strictly decreasing on $[1 / \sqrt{2}, 1)$.

(2) The function $g(r) \equiv \mathcal{K}(r) / r$ is strictly decreasing on $(0,1 / \sqrt{2}]$.

Proof. (1) Differentiation gives

$$
r^{\prime} f^{\prime}(r)=\mathcal{K}^{\prime} \varepsilon-\mathcal{K} \varepsilon^{\prime},
$$

which is strictly decreasing from $(0,1)$ onto $(-\infty, \infty)$ and has a unique zero at $r=1 / \sqrt{2}$. Hence the result for $f$ follows.

(2) By differentiation we obtain

$$
\left(r r^{\prime}\right)^{2} g^{\prime}(r)=\left(\varepsilon-r^{\prime 2} \mathcal{K}\right)-r^{\prime 2} \mathcal{K} \equiv g_{1}(r),
$$

which is strictly increasing from $(0,1)$ onto $(-\pi / 2,1)$ by [7, Theorem $3.21(1),(7)]$, with $g_{1}(1 / \sqrt{2})=\mathcal{E}(1 / \sqrt{2})-\mathcal{K}(1 / \sqrt{2})<0$. Hence the result for $g$ follows.

2.2. Lemma. (1) The function $f(r) \equiv(2 / \pi) \mathcal{K}(r) \mathcal{K}^{\prime}(r)+\log r-\mu\left(r^{\prime}\right)$ is strictly decreasing from $(0,1)$ onto $(0, \log 4)$.

(2) The function $g(r) \equiv(2 / \pi) \mathcal{K}(r) \mathcal{K}^{\prime}(r)+\log \left(r^{\prime} / r\right)$ is strictly decreasing from $(0,1)$ onto $(\log 4, \infty)$.

Proof. (1) Since $f(r)$ can be written as $f(r)=(m(r)+\log r)+\left(m\left(r^{\prime}\right)+\log r^{\prime}\right)-$ $\left(\mu\left(r^{\prime}\right)+\log r^{\prime}\right)$, it follows from [7, Theorem $\left.3.30(1)\right]$ and $[20,(2.11)]$ that $f\left(0^{+}\right)=\log 4$ and $f\left(1^{-}\right)=0$.

From (1.24) and (1.19) we have the formula

$$
\frac{d}{d r}(m(r)+\log r)=-\frac{4}{\pi r} \mathcal{K}^{\prime}(\mathcal{K}-\varepsilon) .
$$

Then, differentiating and using (1.19), (1.20), and (2.3), we get

$$
\frac{\pi}{4} r\left(r^{\prime} \mathcal{K}^{\prime}\right)^{2} f^{\prime}(r)=f_{1}(r) \equiv \mathcal{K}^{\prime 3}\left(\varepsilon-r^{\prime 2} \mathcal{K}\right)-\frac{\pi}{4}\left(r^{2} \mathcal{K}^{\prime 2}+\frac{\pi^{2}}{4}\right) .
$$


It follows from $[7$, Theorem $3.21(1)]$ that

$$
\left\{\begin{aligned}
f_{1}(r)= & r^{2} \mathcal{K}^{\prime 2}\left(\mathcal{K}^{\prime} \frac{\mathcal{E}-r^{\prime 2} \mathcal{K}}{r^{2}}-\frac{\pi}{4}\right)-\frac{\pi^{3}}{16} \\
& <\left(r \mathcal{K}^{\prime}\right)^{2}\left(\mathcal{K}^{\prime}-\frac{\pi}{4}\right)-\frac{\pi^{3}}{16} \equiv f_{2}(r) .
\end{aligned}\right.
$$

Differentiation gives

$$
\frac{r^{\prime 2} f_{2}^{\prime}(r)}{r \mathcal{K}^{\prime 2}\left(\mathcal{K}^{\prime}-\mathcal{E}^{\prime}\right)}=f_{3}(r) \equiv 2-\frac{\pi}{2 \mathcal{K}^{\prime}}-\frac{\mathcal{E}^{\prime}-r^{2} \mathcal{K}^{\prime}}{\mathcal{K}^{\prime}-\mathcal{E}^{\prime}} .
$$

Since $\left(\varepsilon^{\prime}-r^{2} \mathcal{K}^{\prime}\right) /\left(\mathcal{K}^{\prime}-\varepsilon^{\prime}\right)$ is strictly increasing from $(0,1)$ onto $(0,1)[28$, Theorem 1.8 $(2)], f_{3}$ is strictly decreasing from $(0,1)$ onto $(0,2)$, so that $f_{2}$ is strictly increasing on $(0,1)$. Clearly $f_{2}(1)=0$. Hence the monotonicity of $f$ follows from (2.4) and (2.5).

(2) By differentiation and (1.19), we get

$$
g^{\prime}(r)=-\frac{4 \mathcal{K}}{\pi r r^{\prime 2}}\left(\mathcal{E}^{\prime}-r^{2} \mathcal{K}^{\prime}\right)
$$

which is negative by [7, Theorem $3.21(1)]$. Hence the monotonicity of $g$ follows.

Since $g(r)=(m(r)+\log r)+\left(m\left(r^{\prime}\right)+\log r^{\prime}\right)-2 \log r$, the limiting values of $g$ follow from [7, Theorem $3.30(1)]$.

\section{Properties of $\lambda(K)$.}

In this section, we prove several refinements of some known results for the function $\lambda(K)$. Our first result improves [7, Corollary 10.33, Theorem 10.35] and [37, Corollary 2.11].

3.1. Theorem. (1) The function $f(K) \equiv(\log \lambda(K)) /(K-1)$ is strictly decreasing and convex from $(1, \infty)$ onto $(\pi, a)$, where $a=(4 / \pi) \mathcal{K}(1 / \sqrt{2})^{2}=4.3768 \ldots$

(2) The function $g(K) \equiv(1 /(K-1)) \log \left(\lambda(K) e^{b(-K+1 / K)}\right)$, where $b=a / 2$, is strictly increasing from $(1, \infty)$ onto $(0, \pi-b)$.

Proof. Let $r=\mu^{-1}(\pi /(2 K))$. Then, by (1.1), (1.5), and (1.22), $\lambda(K)=\left(r / r^{\prime}\right)^{2}$. Using (1.20), we get

$$
\frac{d r}{d K}=\frac{2}{\pi} r r^{\prime 2} \mathcal{K}^{\prime}(r)^{2}, \quad \frac{d \lambda(K)}{d K}=\frac{4}{\pi} \lambda(K) \mathcal{K}^{\prime}(r)^{2} .
$$

(1) By differentiation and (3.2), we have

$$
f^{\prime}(K)=f_{1}(K) / f_{2}(K)
$$

where $f_{1}(K)=\frac{4}{\pi}(K-1) \mathcal{K}^{\prime}(r)^{2}-\log \lambda(K)$ and $f_{2}(K)=(K-1)^{2}$,

$$
f_{1}^{\prime}(K)=-\left(\frac{4}{\pi}\right)^{2}(K-1) \mathcal{K}^{\prime}(r)^{3}\left[\mathcal{E}^{\prime}(r)-r^{2} \mathcal{K}^{\prime}(r)\right]
$$


and

$$
f_{1}^{\prime}(K) / f_{2}^{\prime}(K)=-\frac{8}{\pi^{2}} \mathcal{K}^{\prime}(r)^{3}\left[\mathcal{E}^{\prime}(r)-r^{2} \mathcal{K}^{\prime}(r)\right] \equiv f_{3}(r) .
$$

From (3.4) and [7, Theorem $3.21(1)]$, we see that $f_{1}$ is strictly decreasing on $(1, \infty)$ with $f_{1}(1)=0$, so that, by $(3.3), f$ is strictly decreasing on $(1, \infty)$.

By [7, Theorem 3.21 (1)], $f_{3}$ is strictly increasing on $(0,1)$, so that $f_{1}^{\prime}(K) / f_{2}^{\prime}(K)$ is strictly increasing in $K$ on $(1, \infty)$, and so is $f^{\prime}$ by the l'Hôpital Monotone Rule [7, Theorem 1.25]. This yields the convexity of $f$.

The limiting values of $f$ follow from [37, Corollary 2.11].

(2) Let $g_{1}(K)=\log \lambda(K)+b\left(\frac{1}{K}-K\right)$ and $g_{2}(K)=K-1$. Then, by (3.2) and differentiation, since $K=\mathcal{K}(r) / \mathcal{K}^{\prime}(r)$, we have

$$
\begin{gathered}
g_{1}^{\prime}(K) / g_{2}^{\prime}(K)=g_{3}(K) \equiv \frac{4}{\pi} \mathcal{K}^{\prime}(r)^{2}-b\left(1+K^{-2}\right), \\
\frac{1}{2} K^{3} g_{3}^{\prime}(K)=b-\frac{8}{\pi^{2}} \mathcal{K}(r)^{3}\left[\mathcal{E}^{\prime}(r)-r^{2} \mathcal{K}^{\prime}(r)\right] \equiv g_{4}(r) .
\end{gathered}
$$

It follows from $[7$, Theorem $3.21(1),(7)]$ that $g_{4}$ is strictly increasing on $(0,1)$. Since $r \in(1 / \sqrt{2}, 1)$ and since, by $(1.19)$,

$$
\left\{\begin{aligned}
g_{4}(1 / \sqrt{2}) & =\frac{2}{\pi} b\left[\frac{\pi}{2}-2 \mathcal{K}(1 / \sqrt{2})\left(\mathcal{E}(1 / \sqrt{2})-\frac{1}{2} \mathcal{K}(1 / \sqrt{2})\right)\right] \\
& =\frac{2}{\pi} b\left[\frac{\pi}{2}-2 \mathcal{K}(1 / \sqrt{2}) \mathcal{E}(1 / \sqrt{2})+\mathcal{K}(1 / \sqrt{2})^{2}\right]=0
\end{aligned}\right.
$$

it follows from $(3.6)$ that $g_{3}$ is strictly increasing on $(1, \infty)$. Hence the monotonicity of $g$ follows from (3.5) and [7, Theorem 1.25].

The limiting values of $g$ follow from l'Hôpital's Rule and (3.5).

3.7. Corollary. For $K>1$,

$$
\max \left\{e^{\pi(K-1)}, e^{b(K-1 / K)}\right\}<\lambda(K)<\min \left\{e^{a(K-1)}, e^{(\pi+b / K)(K-1)}\right\},
$$

where $a$ and $b$ are as in Theorem 3.1. Moreover,

$$
\lim _{K \rightarrow 1} \lambda(K)^{1 /(K-1)}=e^{a}, \quad \lim _{K \rightarrow \infty} \lambda(K)^{1 / K}=e^{\pi} .
$$

Proof. The estimates in (3.8) follow immediately from Theorem 3.1. The lower estimates and first upper estimate in (3.8) also follow from [7, Corollary 10.33, Theorem 10.35], while (3.9) follows directly from (3.8).

In $[8$, Lemma 12, p. 80], P. P. Belinski gave the inequality

$$
\lambda(K)<1+12(K-1)
$$

for $K>1$ close to 1 . However, his proof given in [8, pp. 80-82] for (3.10) is not valid (cf. [37, p. 412]). Corollary 3.5 of [37] gives an improved form of (3.10). Theorem 1.11 is related to this kind of property of $\lambda(K)$ for $K>1$ close to 1 , and improves [37, Corollary 3.5]. 
3.11. Proof of Theorem 1.11. Let $r=\mu^{-1}(\pi /(2 K)), \quad \mathcal{K}=\mathcal{K}(r), \quad \mathcal{K}^{\prime}=\mathcal{K}^{\prime}(r)$, and $\mathcal{E}^{\prime}=\mathcal{E}^{\prime}(r)$. Then (3.2) holds, and by (1.18) and (3.2) we have

$$
\left\{\begin{aligned}
\frac{d \mathcal{K}^{\prime}}{d K} & =-\frac{2}{\pi} \mathcal{K}^{\prime 2}\left(\mathcal{E}^{\prime}-r^{2} \mathcal{K}^{\prime}\right) \\
\lambda^{\prime \prime}(K) & =\frac{4}{\pi} \lambda^{\prime}(K) \mathcal{K}^{\prime}\left[\mathcal{K}^{\prime}-\left(\mathcal{E}^{\prime}-r^{2} \mathcal{K}^{\prime}\right)\right] \\
\lambda^{\prime \prime \prime}(K) & =\frac{6}{\pi} \lambda^{\prime \prime}(K) \mathcal{K}^{\prime}\left[\mathcal{K}^{\prime}-\left(\mathcal{E}^{\prime}-r^{2} \mathcal{K}^{\prime}\right)\right]-\frac{8}{\pi^{2}}\left(1-r^{2} r^{\prime 2}\right) \lambda^{\prime}(K) \mathcal{K}^{\prime 4}
\end{aligned}\right.
$$

By (1.19), we have

$$
\mathcal{K}(1 / \sqrt{2})\left[\mathcal{E}(1 / \sqrt{2})-\frac{1}{2} \mathcal{K}(1 / \sqrt{2})\right]=\frac{\pi}{4} .
$$

Using (3.2), (3.12), and (3.13), we obtain

$$
\lambda^{\prime}(1)=a, \quad \lambda^{\prime \prime}(1)=a(a-1), \quad \lambda^{\prime \prime \prime}(1)=6 c .
$$

Next, let $g(K)=\lambda(K)-1-a(K-1)-\frac{1}{2} a(a-1)(K-1)^{2}$ and $h(K)=(K-1)^{3}$. Then

$$
\left\{\begin{array}{l}
\frac{g^{\prime}(K)}{h^{\prime}(K)}=\frac{\lambda^{\prime}(K)-a-a(a-1)(K-1)}{3(K-1)^{2}} \\
\frac{g^{\prime \prime}(K)}{h^{\prime \prime}(K)}=\frac{\lambda^{\prime \prime}(K)-a(a-1)}{6(K-1)}, \frac{g^{\prime \prime \prime}(K)}{h^{\prime \prime \prime}(K)}=\frac{1}{6} \lambda^{\prime \prime \prime}(K) .
\end{array}\right.
$$

From $(3.2),(3.12)$, and the fact that $\lambda(K)=\left(r / r^{\prime}\right)^{2}$, where $r=\mu^{-1}(\pi /(2 K))$, it follows that

$$
\lambda^{\prime \prime \prime}(K)=\frac{32}{\pi^{3}}\left(r \mathcal{K}^{\prime 2}\right)^{2} F(K)
$$

where

$$
\begin{aligned}
F(K) & =\frac{3}{r^{\prime 2}}\left[\mathcal{K}^{\prime}-\left(\mathcal{E}^{\prime}-r^{2} \mathcal{K}^{\prime}\right)\right]^{2}-\frac{1-r^{2} r^{\prime 2}}{r^{\prime 2}} \mathcal{K}^{\prime 2} \\
& =2\left(\frac{\mathcal{K}^{\prime}}{r^{\prime}}\right)^{2}+\left(r \mathcal{K}^{\prime}\right)^{2}-3 \frac{\mathcal{E}^{\prime}-r^{2} \mathcal{K}^{\prime}}{r^{\prime 2}}\left[\left(\mathcal{K}^{\prime}+\mathcal{E}^{\prime}\right)+\left(1+r^{2}\right) \mathcal{K}^{\prime}-2 \mathcal{E}^{\prime}\right] .
\end{aligned}
$$

Using (3.13), we get

$$
\begin{aligned}
F(1) & =6\left\{\mathcal{K}(1 / \sqrt{2})-\left[\mathcal{E}(1 / \sqrt{2})-\frac{1}{2} \mathcal{K}(1 / \sqrt{2})\right]\right\}^{2}-\frac{3}{2} \mathcal{K}(1 / \sqrt{2})^{2} \\
& =\frac{9}{2} \mathcal{K}(1 / \sqrt{2})^{2}+6\left[\mathcal{E}(1 / \sqrt{2})-\frac{1}{2} \mathcal{K}(1 / \sqrt{2})\right]^{2}-3 \pi=7.1210 \cdots>0 .
\end{aligned}
$$

Since $r \in(1 / \sqrt{2}, 1)$, it follows from Lemma 2.1 (2) and [7, Theorem 3.21(7)], that $\mathcal{K}^{\prime} / r^{\prime}$ and $r \mathcal{K}^{\prime}$ are increasing, while by [7, Theorem $3.21(1)$, Lemma $\left.1.33(3)\right]\left(\mathcal{E}^{\prime}-r^{2} \mathcal{K}^{\prime}\right) / r^{\prime 2}$ and $\mathcal{K}^{\prime}+\mathcal{E}^{\prime}$ are decreasing. Next, by [7, Exercise $\left.3.43(4)(\mathrm{a})\right],\left(1+r^{2}\right) \mathcal{K}^{\prime}-2 \mathcal{E}^{\prime}$ is strictly 
decreasing from $(0,1)$ onto $(0, \infty)$. Thus we conclude that $F(K)$ is strictly increasing in $K$ on $(1, \infty)$. Hence, it follows from [7, Theorem 3.21 (7)] and (3.16) that $\lambda^{\prime \prime \prime}$ is strictly increasing on $(1, \infty)$.

By (3.14), we observe that $g^{\prime}(1)=h^{\prime}(1)=g^{\prime \prime}(1)=h^{\prime \prime}(1)=0$. Hence the monotonicity of $f$ follows from (3.15) and [7, Theorem 1.25].

By l'Hôpital's Rule, (3.14), and (3.15), we get $f\left(1^{+}\right)=c$. Since $K=\mathcal{K} / \mathcal{K}^{\prime}, \quad \lambda(K)=$ $\left(r / r^{\prime}\right)^{2}$, and

$$
f(K)=G(r)=\frac{r^{2}-r^{\prime 2}\left\{1+a\left[\left(\mathcal{K} / \mathcal{K}^{\prime}\right)-1\right]+a_{1}\left[\left(\mathcal{K} / \mathcal{K}^{\prime}\right)-1\right]^{2}\right\}}{r^{\prime 2}\left[\left(\mathcal{K} / \mathcal{K}^{\prime}\right)-1\right]^{3}},
$$

where $a_{1}=\frac{1}{2} a(a-1)$, and since $\sqrt{r^{\prime}} \mathcal{K} \rightarrow 0$ as $r \rightarrow 1[7$, Theorem 3.21(7)], we see that

$$
\lim _{K \rightarrow \infty} f(K)=\lim _{r \rightarrow 1} G(r)=\infty .
$$

Finally, (1.12) is clear. Since

$$
f(2)=\lambda(2)-1-a-\frac{1}{2} a(a-1)=c_{1},
$$

(1.13) follows from the monotonicity of $f$. We have used [7, Theorem 10.5(4)] in the evaluation of $\lambda(2)$.

3.17. Corollary. (1) As $K \rightarrow 1$,

$$
\lambda(K)=1+a(K-1)+\frac{1}{2} a(a-1)(K-1)^{2}+O\left((K-1)^{3}\right),
$$

where $a$ is as in Theorem 1.11 .

(2) Let $\delta>0$ be an arbitrary real number and let $c_{1}$ be as in Theorem 1.11. Then, for $1<K \leq K_{0} \equiv 1+\left(\sqrt{a_{1}^{2}+4 c_{1} \delta}-a_{1}\right) /\left(2 c_{1}\right)$,

$$
\lambda(K)<1+(a+\delta)(K-1)
$$

In particular, for

$$
\begin{gathered}
1<K \leq 1+\left(\sqrt{a_{1}^{2}+4 c_{1}(5-a)}-a_{1}\right) /\left(2 c_{1}\right)=1.07066 \ldots, \\
\lambda(K)<1+5(K-1) .
\end{gathered}
$$

Proof. Part (1) follows immediately from (1.13). By (1.13), we see that (3.18) holds if

$$
c_{1}(K-1)^{2}+a_{1}(K-1)-\delta \leq 0 .
$$

Clearly, for $K>1,(3.20)$ holds if and only if $K \leq K_{0}$.

Taking $\delta=5-a$, we obtain (3.19) from (3.18).

The next result improves (1.10). 
3.21. Theorem. For $K>1$,

$$
\frac{1}{16} e^{\pi K}-\frac{1}{2}+c_{1}(K) e^{-\pi K}<\lambda(K)<\frac{1}{16} e^{\pi K}-\frac{1}{2}+c_{2}(K) e^{-\pi K},
$$

where

$$
\begin{gathered}
c_{1}(K) \equiv \frac{1}{4}+\frac{1}{4 e^{-2 \pi K}+1}>\frac{21}{16}-c, \\
c_{2}(K) \equiv \frac{1}{16}\left(1+4 \frac{5 e^{-4 \pi K}+14 e^{-2 \pi K}+5}{e^{-6 \pi K}+7 e^{-4 \pi K}+7 e^{-2 \pi K}+1}\right)<\frac{21}{16},
\end{gathered}
$$

and $c \equiv\left(68+e^{2 \pi}\right) /\left[16\left(4+e^{2 \pi}\right)\right]=0.06991 \ldots$

Proof. It follows from $[7,(5.20),(5.4)$, Theorem 5.13 (4)] that, for all $r \in(0,1)$,

$$
\log \frac{1+\sqrt[4]{r^{\prime}}}{1-\sqrt[4]{r^{\prime}}}<2 \mu(r)<\log \frac{2\left(1+\sqrt{r^{\prime}}\right)}{1-\sqrt{r^{\prime}}}
$$

from which we get, with $x \equiv \exp (2 \mu(r)) \geq 2$,

$$
1-\left(\frac{x-1}{x+1}\right)^{8}<r^{2}<1-\left(\frac{x-2}{x+2}\right)^{4}
$$

for all $r \in\left(0, \mu^{-1}(\log \sqrt{2})\right]$.

Let $r=\mu^{-1}(\pi K / 2)$. Then $0<r<1 / \sqrt{2}<\mu^{-1}(\log \sqrt{2}), \quad x=e^{\pi K}$, and $\lambda(K)=\left(r^{\prime} / r\right)^{2}$.

It follows from the second inequality in (3.24) that

$$
\begin{aligned}
\lambda(K) & =\frac{1}{r^{2}}-1>\frac{(x+2)^{4}}{16 x\left(x^{2}+4\right)}-1=\frac{e^{\pi K}}{16} \frac{\left(1+2 e^{-\pi K}\right)^{4}}{1+4 e^{-2 \pi K}}-1 \\
& =\frac{e^{\pi K}}{16}\left[1+8 e^{-\pi K}+4 e^{-2 \pi K}\left(1+\frac{4}{4 e^{-2 \pi K}+1}\right)\right]-1 \\
& =\frac{1}{16} e^{\pi K}-\frac{1}{2}+\frac{1}{4} e^{-\pi K}\left(1+\frac{4}{4 e^{-2 \pi K}+1}\right),
\end{aligned}
$$

and hence the first inequality in (3.22) holds. Clearly,

$$
c_{1}(K)>\frac{1}{4}+\frac{1}{4 e^{-2 \pi}+1}=\frac{21}{16}-c .
$$

Next, it follows from the first inequality in (3.24) that

$$
\begin{aligned}
\lambda(K) & =\frac{1}{r^{2}}-1<\frac{x\left(1+x^{-1}\right)^{8}}{16\left(1+x^{-2}\right)\left(x^{-4}+6 x^{-2}+1\right)}-1 \\
& =\frac{1}{16} e^{\pi K}+\frac{e^{\pi K}}{16} \frac{(1+y)^{8}-\left(1+y^{2}\right)\left(1+6 y^{2}+y^{4}\right)}{\left(1+y^{2}\right)\left(1+6 y^{2}+y^{4}\right)}-1 \\
& =\frac{1}{16} e^{\pi K}+\frac{e^{\pi K}}{16}\left[8 y+y^{2}\left(1+4 \frac{5 y^{4}+14 y^{2}+5}{y^{6}+7 y^{4}+7 y^{2}+1}\right)\right]-1,
\end{aligned}
$$


where $y=1 / x=e^{-\pi K}$, and hence the second inequality in (3.22) follows.

Finally, it is easy to verify that $\left(5 t^{2}+14 t+5\right) /\left(t^{3}+7 t^{2}+7 t+1\right)$ is a strictly decreasing function of $t$ on $(0, \infty)$. Hence $c_{2}(K)$ is strictly increasing on $[1, \infty)$, so that $c_{2}(K)<$ $\lim _{K \rightarrow \infty} c_{2}(K)=21 / 16$.

3.25. Remark. Computation gives:

$$
\begin{aligned}
\frac{1}{16} e^{\pi}-\frac{1}{2}+c_{1}(1) e^{-\pi} & =0.9999902 \ldots \\
\frac{1}{16} e^{\pi}-\frac{1}{2}+c_{2}(1) e^{-\pi} & =1.0025922 \ldots
\end{aligned}
$$

Hence, even when $K$ is close to 1 , the lower and upper estimates for $\lambda(K)$ given in $(3.22)$ are very close to each other.

\section{Properties of $\eta_{K}(t)$.}

In this section, we study some properties of $\eta_{K}(t)$. We first extend to $\eta_{K}(t)$ the properties of $\lambda(K)$ proved in [32, Lemma 3.54].

4.1. Proof of Theorem 1.14. Let $s=\varphi_{K}(r)$. Then $\eta_{K}(t)=\left(s / s^{\prime}\right)^{2}$ and, using $(1.4)$ and (1.21), we have

$$
\frac{\partial \eta_{K}(t)}{\partial K}=\frac{4}{\pi K} \eta_{K}(t) \mathcal{K}(s) \mathcal{K}^{\prime}(s)=\frac{2}{\mu(r)} \mathcal{K}^{\prime}(s)^{2} \eta_{K}(t) .
$$

(1) We may rewrite $f(K)$ as

$$
f(K)=\left[\frac{s}{s^{\prime} e^{\mu\left(s^{\prime}\right)}}\right]^{2}=e^{-2\left[\mu\left(s^{\prime}\right)+\log \left(s^{\prime} / s\right)\right]} .
$$

Hence the monotonicity of $f$ follows from [7, Theorem 5.13 (3)]. The limiting values are clear.

(2) By (4.2) we may write $g(K)$ as

$$
g(K)=\frac{2 \mathcal{K}^{\prime}(s)^{2}}{\mu(r)}
$$

from which the monotonicity follows immediately. We obtain (1.15) by integrating the inequalities

$$
2 \mu\left(r^{\prime}\right)<g(K)<\frac{4}{\pi} \mathcal{K}(r) \mathcal{K}^{\prime}(r)
$$

with respect to $K$ over $(1, K)$ and using the fact that $\eta_{1}(t)=t$.

(3) By (4.2), we have

$$
h(K)=\frac{2}{\mu(r)}\left[\frac{\sqrt{s} \mathcal{K}^{\prime}(s)}{\left(s^{\prime} / \sqrt{s}\right) e^{\mu\left(s^{\prime}\right)}}\right]^{2} .
$$


Hence the monotonicity of $h$ follows from [7, Theorem 3.21(7)] and [32, Lemma 3.54(1)]. We obtain (1.16) and (1.17) by integrating the inequalities

$$
\frac{4}{\pi} t \mathcal{K}(r) \mathcal{K}^{\prime}(r) e^{2(K-1) \mu\left(r^{\prime}\right)}<\frac{\partial \eta_{K}}{\partial K}<\frac{1}{8} \mu\left(r^{\prime}\right) e^{2 K \mu\left(r^{\prime}\right)}
$$

with respect to $K$ over $(1, K)$.

4.3. Remark. An injective mapping $f: X \rightarrow Y$, where $X$ and $Y$ are metric spaces with distances denoted by $|a-b|$, is called quasisymmetric if there exists a homeomorphism $\eta:[0, \infty) \rightarrow[0, \infty), \eta(0)=0$, such that for all $a, b, c \in X, a \neq c$,

$$
\frac{|f(a)-f(b)|}{|f(a)-f(c)|} \leq \eta\left(\frac{|a-b|}{|a-c|}\right) .
$$

Further, if $s>0$, we say that $f$ is s-quasisymmetric if $f$ is quasisymmetric with $\eta(t) \leq t+s$ for all $t \in(0, \max \{1,1 / s\})$. Using (1.16), one can show that, for $K$ sufficiently close to 1 , a $K$-quasiconformal map $f$ of $\mathbb{R}^{2}$ into $\mathbb{R}^{2}$ is $s$-quasisymmetric with $s=(K-1)^{4 / 9}$. This result improves the conclusion stated in [36, Remark 2.17], where it was indicated that $f$ is $s$-quasisymmetric with $s=\sqrt[4]{K-1}$. In fact, by [36, Theorem 2.16] and (1.16), we only need to show that, for $K$ sufficiently close to 1 ,

$$
e^{2 \mu(r)}\left[e^{2(K-1) \mu(r)}-1\right] / s \leq 16,
$$

where $r=\sqrt{s /(1+s)}$ and $s=(K-1)^{4 / 9}$. Since $K=1+s^{9 / 4}$ and $s=\left(r / r^{\prime}\right)^{2}$, it follows from [20, (2.11), p. 62], [7, Theorem 3.21 (7)], l'Hôpital's Rule, and (1.20) that

$$
\begin{aligned}
\lim _{K \rightarrow 1} e^{2 \mu(r)}\left[e^{2(K-1) \mu(r)}-1\right] / s \\
\quad=\lim _{r \rightarrow 0} e^{2(\mu(r)+\log r)} \frac{e^{2\left(r / r^{\prime}\right)^{9 / 2} \mu(r)}-1}{r^{4}}=16 \lim _{r \rightarrow 0} \frac{e^{2\left(r / r^{\prime}\right)^{9 / 2} \mu(r)}-1}{r^{4}} \\
\quad=\frac{8}{\pi} \lim _{r \rightarrow 0} \sqrt{r}\left[9 \mathcal{K}(r) \mathcal{K}^{\prime}(r)-\pi\right]=0 .
\end{aligned}
$$

Thus there exists $K_{0} \in(1,2)$ such that (4.4) holds for $K \in\left(1, K_{0}\right]$.

The next result extends [6, Theorem 2.3] to the function $\eta_{K}(t)$ for $K>0$.

4.5. Theorem. For each fixed $t \in(0, \infty), F(K) \equiv \eta_{K}(t)+1$ is log-convex as a function of $K$ on $(0, \infty)$, while $\eta_{K}(t)$ is log-concave there. In particular, for $t, K, L \in(0, \infty)$ and $p, q \in(0,1)$ with $p+q=1$,

$$
\eta_{K}(t)^{p} \eta_{L}(t)^{q}<\eta_{p K+q L}(t)<\left[\eta_{K}(t)+1\right]^{p}\left[\eta_{L}(t)+1\right]^{q}-1 .
$$

Proof. First, we observe that (4.2) also holds for $K \in(0,1)$, so that the monotonicity properties of $f, g$, and $h$ in Theorem 1.14 are valid for $K \in(0, \infty)$. Hence the log-concavity of $\eta_{K}(t)$ follows from Theorem 1.14 (2). Alternatively, the logarithmic derivative of $\eta_{K}(t)$ is $(2 / \mu(r)) \mathcal{K}^{\prime}(s)^{2}$, which is strictly decreasing in $K$. 
Since $\eta_{K}(t)=\left(s / s^{\prime}\right)^{2}$, where $s=\varphi_{K}(r)$ and $r=\sqrt{t /(1+t)}$, we have

$$
F(K)=\eta_{K}(t)+1=\left(s^{\prime}\right)^{-2}
$$

and hence, by (1.21),

$$
\frac{F^{\prime}(K)}{F(K)}=\frac{2}{\mu(r)} s^{2} \mathcal{K}^{\prime}(s)^{2},
$$

which is strictly increasing in $K$ on $(0, \infty)$ by [7, Theorem $3.21(7)]$. The log-convexity of $F$ now follows.

The remaining conclusions are clear.

The next result is a generalization of Theorem 3.21 to the function $\eta_{K}(t)$, and provides an analogue of (1.10) for $\eta_{K}(t)$.

4.7. Theorem. (1) For $K>1$ and $0<t<\left[\mu^{-1}\left(\frac{1}{2 K} \log 2\right)\right]^{-2}-1$,

$$
\eta_{K}(t)<\left[\mu^{-1}\left(\frac{1}{2} \log 2\right)\right]^{-2}-1<0.000011 .
$$

(2) For $K>1$ and $t \geq\left[\mu^{-1}\left(\frac{1}{2 K} \log 2\right)\right]^{-2}-1$,

$$
\eta_{K}(t)>\frac{1}{16} e^{2 K \mu\left(r^{\prime}\right)}-\frac{1}{2}+c_{1}(t, K) e^{-2 K \mu\left(r^{\prime}\right)},
$$

where $r=\sqrt{t /(1+t)}$ and

$$
c_{1}(t, K)=\frac{1}{4}+\frac{1}{4 e^{-4 K \mu\left(r^{\prime}\right)}+1}>\frac{1}{4}+\frac{1}{4 e^{-4 \mu\left(r^{\prime}\right)}+1}>\frac{9}{20} .
$$

(3) For $t \in(0, \infty)$ and $K>1$,

$$
\eta_{K}(t)<\frac{1}{16} e^{2 K \mu\left(r^{\prime}\right)}-\frac{1}{2}+c_{2}(t, K) e^{-2 K \mu\left(r^{\prime}\right)},
$$

where $r$ is as in part (2) and

$$
\left\{\begin{aligned}
c_{2}(t, K) & =\frac{1}{16}\left[1+4 \frac{5 e^{-8 K \mu\left(r^{\prime}\right)}+14 e^{-4 K \mu\left(r^{\prime}\right)}+5}{e^{-12 K \mu\left(r^{\prime}\right)}+7 e^{-8 K \mu\left(r^{\prime}\right)}+7 e^{-4 K \mu\left(r^{\prime}\right)}+1}\right] \\
& <\frac{5}{16}+\frac{1}{4 e^{-2 K \mu\left(r^{\prime}\right)}+1}<\frac{21}{16} .
\end{aligned}\right.
$$

Proof. Let $u=\varphi_{1 / K}\left(r^{\prime}\right), r=\sqrt{t /(1+t)}$, and $x=e^{2 \mu(u)}=e^{2 K \mu\left(r^{\prime}\right)}$. Then, by (1.6) and (1.22),

$$
\eta_{K}(t)=u^{-2}-1
$$

and, by (3.23),

$$
\frac{1+\sqrt[4]{u^{\prime}}}{1-\sqrt[4]{u^{\prime}}}<x<2 \frac{1+\sqrt{u^{\prime}}}{1-\sqrt{u^{\prime}}}
$$


(1) For $K>1$ and $0<t<\left[\mu^{-1}\left(\frac{1}{2 K} \log 2\right)\right]^{-2}-1$, we have

$$
u=\varphi_{1 / K}\left(r^{\prime}\right)>\mu^{-1}\left(K \mu\left(\mu^{-1}\left(\frac{1}{2 K} \log 2\right)\right)\right)=\mu^{-1}\left(\frac{1}{2} \log 2\right)
$$

so that, by (4.13),

$$
\eta_{K}(t)<\left[\mu^{-1}\left(\frac{1}{2} \log 2\right)\right]^{-2}-1<0.99999476^{-2}-1<0.000011 .
$$

(2) Since $t \geq\left[\mu^{-1}\left(\frac{1}{2 K} \log 2\right)\right]^{-2}-1$, we have

$$
x=e^{2 K \mu(1 / \sqrt{1+t})} \geq e^{2 K \mu\left(\mu^{-1}\left(\frac{1}{2 K} \log 2\right)\right)}=2 .
$$

Hence, it follows from the second inequality in (4.14) that

$$
u^{2}<1-\left(\frac{x-2}{x+2}\right)^{4}
$$

so that, by (4.13),

$$
\eta_{K}(t)>\frac{(x+2)^{4}}{16 x\left(x^{2}+4\right)}-1 .
$$

Then, by the method used in the proof of Theorem 3.21 one can easily obtain (4.9). The inequalities in (4.10) are clear.

(3) Clearly, $x>1$ for all $t>0$ and $K>1$. Hence, it follows from the first inequality in (4.14) that

$$
u^{2}>1-\left(\frac{x-1}{x+1}\right)^{8} .
$$

Now, using (4.13), one can prove (4.11) by the method used in the proof of the second inequality in (3.22), without any difficulty.

Finally, let

$$
f(y)=4 \frac{5 y^{2}+56 y+80}{y^{3}+28 y^{2}+112 y+64}-\frac{4}{y+1}-\frac{3}{4} .
$$

Then $c_{2}(t, K)$ can be written as

$$
c_{2}(t, K)=\frac{1}{4}\left[1+\frac{4}{b^{2}+1}+f\left(b^{2}\right)\right],
$$

where $b=2 / x=2 e^{-2 K \mu\left(r^{\prime}\right)}$. Clearly, $b \in(0,2)$. It is easy to show that the function $f$, which can be rewritten as

$$
f(y)=4 \frac{4 y^{3}+33 y^{2}+24 y+16}{(y+1)\left(y^{3}+28 y^{2}+112 y+64\right)}-\frac{3}{4},
$$

is strictly decreasing on $(0,4)$ and that $f(0)=1 / 4$. Hence the inequalities in $(4.12)$ follow from (4.15).

As a consequence of Theorem 4.7, the following corollary gives an asymptotic expansion for $\eta_{K}(t)$ as $K \rightarrow \infty$ or $t \rightarrow \infty$. 
4.16. Corollary. As $K \rightarrow \infty$ or $t \rightarrow \infty$,

$$
\eta_{K}(t)=\frac{1}{16} e^{2 K \mu\left(r^{\prime}\right)}-\frac{1}{2}+O\left(e^{-2 K \mu\left(r^{\prime}\right)}\right),
$$

where $r=\sqrt{t /(1+t)}$.

In [32, Theorem 1.3], it was proved that, for $t \in(0, \infty)$ and $K \in(1, \infty)$,

$$
16^{1-1 / K}<\frac{\eta_{K}(t)}{t^{1 / K}(1+t)^{K-1 / K}}<16^{K-1} c^{2(1-1 / K)},
$$

where $c=(1 / 8) \exp \left(2 \mathcal{K}(1 / \sqrt{2})^{2} / \pi\right)=1.115 \ldots$ The proof of (4.17) given in [32, Proof of Theorem 1.3], however, is complicated and long. Our next result improves (4.17), and its proof is also much simpler.

4.18. Theorem. (1) For each $t \in(0, \infty)$, the function

$$
f(K) \equiv 16^{1 / K} \eta_{K}(t) /\left[t^{1 / K}(1+t)^{K-1 / K}\right]
$$

is strictly increasing and convex from $[1, \infty)$ onto $[16, \infty)$. In particular, for $t \in(0, \infty)$ and $K>1$,

$$
\eta_{K}(t)>16^{1-1 / K} t^{1 / K}(1+t)^{K-1 / K},
$$

and, for $t \in(0, \infty)$ and $K \in(1,2)$,

$$
16^{1-1 / K}<\frac{\eta_{K}(t)}{t^{1 / K}(1+t)^{K-1 / K}}<16^{1-1 / K}\left\{1+\left[\left(1+\sqrt{\frac{t}{1+t}}\right)^{2}-1\right](K-1)\right\} .
$$

(2) For $t \in(0, \infty)$, let $r=\sqrt{t /(1+t)}, A=A(r)=r^{\prime} e^{\mu\left(r^{\prime}\right)}$ and $B=B(r)=$ $r \exp \left((2 / \pi) \mathcal{K}(r) \mathcal{K}^{\prime}(r)-\mu\left(r^{\prime}\right)\right)$. Then the function

$$
g(K) \equiv A^{-K} B^{1 / K}\left[\frac{\eta_{K}(t)}{t^{1 / K}(1+t)^{K-1 / K}}\right]^{1 / 2}
$$

is strictly decreasing from $[1, \infty)$ onto $(1 / 4, B / A]$. In particular, for $t \in(0, \infty)$ and $K \in$ $(1, \infty)$

$$
\left\{\begin{aligned}
\frac{1}{16} A^{2 K} B^{-2 / K} & <\frac{\eta_{K}(t)}{t^{1 / K}(1+t)^{K-1 / K}}<A^{2(K-1)} B^{2(1-1 / K)} \\
& \leq 16^{K-1} c^{2(1-1 / K)}
\end{aligned}\right.
$$

where $c$ is as in (4.17).

Proof. Let $r=\sqrt{t /(1+t)}, s=\varphi_{K}(r)$.

For part (1), we rewrite $f$ as

$$
f(K)=\left(4^{1 / K} \frac{s}{s^{\prime}} \frac{r^{\prime K}}{r^{1 / K}}\right)^{2} .
$$


By logarithmic differentiation and (1.21), we obtain

$$
\left\{\begin{aligned}
\frac{1}{2} f^{\prime}(K) & =f(K)\left[\frac{1}{\mu(r)} \mathcal{K}^{\prime}(s)^{2}+\frac{1}{K^{2}} \log \frac{r}{4}+\log r^{\prime}\right] \\
& \equiv f(K) F_{1}(K)
\end{aligned}\right.
$$

Clearly, $F_{1}$ has the following limiting values:

$$
F_{1}(1)=m(r)+m\left(r^{\prime}\right)+\log \frac{r r^{\prime}}{4}, \lim _{K \rightarrow \infty} F_{1}(K)=\mu\left(r^{\prime}\right)+\log r^{\prime} .
$$

Differentiation gives

$$
\frac{1}{2} K^{3} F_{1}^{\prime}(K)=F_{2}(K) \equiv \log \frac{4}{r}-\frac{4}{\pi^{2}} \frac{\mathcal{K}^{\prime}(r)}{\mathcal{K}(r)} \mathcal{K}(s)^{3}\left[\mathcal{E}^{\prime}(s)-s^{2} \mathcal{K}^{\prime}(s)\right] .
$$

It follows from $[7$, Theorem $3.21(1),(7)]$ that $F_{2}$ is strictly increasing on $[1, \infty)$, and

$$
\begin{aligned}
F_{2}(1) & =\left[1-\frac{8}{\pi^{3}} r^{\prime 2} \mathcal{K}(r)^{3} \cdot \frac{\mu(r)}{\log (4 / r)} \cdot \frac{\mathcal{E}^{\prime}(r)-r^{2} \mathcal{K}^{\prime}(r)}{r^{\prime 2}}\right] \log \frac{4}{r}, \\
\lim _{K \rightarrow \infty} F_{2}(K) & =\log \frac{4}{r}
\end{aligned}
$$

From [7, Theorems $3.21(1),(7)$ and $5.16(2)]$, we see that the function $F_{2}(1) / \log (4 / r)$ is strictly increasing in $r$ from $(0,1)$ onto $(0,1)$. Hence, by $(4.24), F_{1}$ is strictly increasing on $[1, \infty)$. Since $F_{1}(1)>0$ for all $r \in(0,1)$ [32, Lemma 2.27], $F_{1}(K)>0$ for all $K \geq 1$ and $r \in(0,1)$. Hence, $f(K) F_{1}(K)$ is positive and increasing on $[1, \infty)$ by $(4.22)$, and the monotonicity and convexity of $f$ follow from (4.22).

Clearly, $f(1)=16$. It follows from [20, (2.11), p. 62] that

$$
\begin{aligned}
\lim _{K \rightarrow \infty} \frac{r^{\prime K}}{s^{\prime}} & =\lim _{K \rightarrow \infty} \frac{\exp \left[\mu\left(s^{\prime}\right)\left(1+\left(\log r^{\prime}\right) / \mu\left(r^{\prime}\right)\right)\right]}{\exp \left(\mu\left(s^{\prime}\right)+\log s^{\prime}\right)} \\
& =\frac{1}{4} \lim _{s \rightarrow 1} \exp \left[\frac{\mu\left(s^{\prime}\right)}{\mu\left(r^{\prime}\right)}\left(\mu\left(r^{\prime}\right)+\log r^{\prime}\right)\right]=\infty
\end{aligned}
$$

and hence $\lim _{K \rightarrow \infty} f(K)=\infty$.

Inequality (4.19) is clear, while the upper bound in (4.20) follows from the convexity of $f$ and (1.22).

For part (2), we write $g$ as

$$
g(K)=\frac{s}{s^{\prime}}\left(\frac{r^{\prime}}{A}\right)^{K}\left(\frac{B}{r}\right)^{1 / K}
$$

Logarithmic differentiation gives

$$
\frac{K^{2}}{g(K)} g^{\prime}(K)=g_{1}(K) \equiv \frac{4}{\pi^{2}} \mu(r) \mathcal{K}(s)^{2}+K^{2} \log \frac{r^{\prime}}{A}+\log \frac{r}{B}
$$


and

$$
\frac{1}{2 K} g_{1}^{\prime}(K)=g_{2}(K) \equiv \frac{4}{\pi^{2}} \frac{\mathcal{K}(r)}{\mathcal{K}^{\prime}(r)} \mathcal{K}^{\prime}(s)^{3}\left[\mathcal{E}(s)-s^{\prime 2} \mathcal{K}(s)\right]+\log \frac{r^{\prime}}{A} .
$$

From [7, Theorem $3.21(1)$, (7)] we see that $g_{2}$ is strictly increasing on $[1, \infty)$ so that, for all $K \geq 1$,

$$
g_{2}(K)<\lim _{K \rightarrow \infty} g_{2}(K)=\mu\left(r^{\prime}\right)+\log \left(r^{\prime} / A\right)=0 .
$$

Hence $g_{1}$ is strictly decreasing on $[1, \infty)$, so that

$$
g_{1}(K)<g_{1}(1)=\frac{2}{\pi} \mathcal{K}(r) \mathcal{K}^{\prime}(r)+\log \left(r r^{\prime} /(A B)\right)=0
$$

for $K>1$. Consequently, the monotonicity of $g$ follows from (4.25).

Clearly, $g(1)=B / A$. From $[20,(2.11)$, p. 62], we get

$$
\lim _{K \rightarrow \infty} g(K)=\lim _{K \rightarrow \infty} \frac{s}{s^{\prime}}\left(\frac{r^{\prime}}{A}\right)^{K}=\lim _{s \rightarrow 1} \frac{1}{s^{\prime} e^{\mu\left(s^{\prime}\right)}}=\frac{1}{4} .
$$

The first and second inequalities in (4.21) are clear. The third inequality in (4.21) holds if and only if $(A / 4)^{K}(B / c) \leq 1$ for all $K>1$ and $t>0$. This is true if and only if $A B /(4 c) \leq 1$ since $A<4$. Now [32, Lemma 2.27] implies that $A B \leq 4 c$.

4.26. Remark. It follows from $[7$, Theorem $5.13(2)]$ and Lemma $2.2(1)$ that $A^{K} B^{-1 / K}$ is strictly increasing in $r$ from $(0,1)$ onto $\left(4^{-1 / K}, 4^{K}\right)$. Hence the lower bound in $(4.21)$ tends to $16^{K-1}$ as $r$ tends to 1 , that is, as $t$ tends to $\infty$. Consequently, for large $t$, the lower bound in (4.21) is better than the lower bound $16^{1-1 / K}$ given in (4.17).

4.27. Theorem. For $t \in(0, \infty)$, let $r=\sqrt{t /(1+t)}$.

(1) The function

$$
f(K) \equiv\left\{\eta_{K}(t) \exp \left(2\left(\frac{2}{\pi} \mathcal{K}(r) \mathcal{K}^{\prime}(r)+\log \frac{r^{\prime}}{r}\right)\right)\right\}^{1 / K}
$$

is strictly decreasing from $[1, \infty)$ onto $\left(e^{2 \mu\left(r^{\prime}\right)}, e^{4 \mathcal{K}(r) \mathcal{K}^{\prime}(r) / \pi}\right]$. In particular, for $t \in(0, \infty)$ and $K \in(1, \infty)$,

$$
t \exp \left\{2\left[K \mu\left(r^{\prime}\right)-\frac{2}{\pi} \mathcal{K}(r) \mathcal{K}^{\prime}(r)\right]\right\}<\eta_{K}(t)<t \exp \left\{\frac{4}{\pi}(K-1) \mathcal{K}(r) \mathcal{K}^{\prime}(r)\right\}
$$

(2) The function

$$
g(K) \equiv \frac{1}{K-1} \log \left\{\frac{\eta_{K}(t)}{t^{1 / K}(1+t)^{K-1 / K}} \exp \left[2\left(\frac{1}{K}-1\right)\left(\frac{2}{\pi} \mathcal{K}(r) \mathcal{K}^{\prime}(r)+\log \left(r r^{\prime}\right)\right)\right]\right\}
$$


is strictly increasing from $(1, \infty)$ onto $\left(0,2\left[\mu\left(r^{\prime}\right)+\log r^{\prime}\right]\right)$. In particular, for $t \in(0, \infty)$ and $K \in(1, \infty)$,

$$
\left\{\begin{aligned}
16^{1-1 / K} & <\exp \left\{2\left(1-\frac{1}{K}\right)\left(\frac{2}{\pi} \mathcal{K}(r) \mathcal{K}^{\prime}(r)+\log \left(r r^{\prime}\right)\right)\right\} \\
& <\frac{\eta_{K}(t)}{t^{1 / K}(1+t)^{K-1 / K}} \\
& <\exp \{2(K-1))\left[\mu\left(r^{\prime}\right)+\log r^{\prime}\right] \\
& \left.+2\left(1-\frac{1}{K}\right)\left[\frac{2}{\pi} \mathcal{K}(r) \mathcal{K}^{\prime}(r)+\log \left(r r^{\prime}\right)\right]\right\}
\end{aligned}\right.
$$

and

$$
e^{(K-1)[\log 2+(a-\log 2) / K]}<\lambda(K)<e^{(K-1)[\pi+(a-\log 2) / K]},
$$

where $a=(4 / \pi) \mathcal{K}(1 / \sqrt{2})^{2}$.

Proof. Let $s=\varphi_{K}(r)$. Then $\eta_{K}(t)=\left(s / s^{\prime}\right)^{2}$.

(1) Logarithmic differentiation gives

$$
\frac{K^{2}}{2 f(K)} f^{\prime}(K)=f_{1}(s)-f_{1}(r)
$$

where $f_{1}(x)=(2 / \pi) \mathcal{K}(x) \mathcal{K}^{\prime}(x)+\log \left(x^{\prime} / x\right)$. Hence the monotonicity of $f$ follows from Lemma $2.2(2)$.

Clearly, $f(1)=e^{4 \mathcal{K}(r) \mathcal{K}^{\prime}(r) / \pi}$. By l'Hôpital's Rule, (4.2), and [7, (5.2)], we have

$$
\lim _{K \rightarrow \infty} \eta_{K}(t)^{1 / K}=\exp \left(\lim _{K \rightarrow \infty} \frac{\log \eta_{K}(t)}{K}\right)=e^{2 \mu\left(r^{\prime}\right)} .
$$

Hence $\lim _{K \rightarrow \infty} f(K)=e^{2 \mu\left(r^{\prime}\right)}$.

(2) Let

$$
g_{1}(K)=\log \left(s / s^{\prime}\right)+K \log r^{\prime}+((1 / K)-1)\left[(2 / \pi) \mathcal{K}(r) \mathcal{K}^{\prime}(r)+\log r^{\prime}\right]-\log r
$$

and $g_{2}(K)=K-1$. Then $g(K)=2 g_{1}(K) / g_{2}(K)$ and, by differentiation,

$$
\frac{g_{1}^{\prime}(K)}{g_{2}^{\prime}(K)}=g_{3}(K) \equiv \frac{1}{\mu(r)} \mathcal{K}^{\prime}(s)^{2}-\frac{1}{K^{2}}\left[\frac{2}{\pi} \mathcal{K}(r) \mathcal{K}^{\prime}(r)+\log r^{\prime}\right]+\log r^{\prime}
$$

and

$$
\left\{\begin{aligned}
& \frac{K^{3}}{2} g_{3}^{\prime}(K)=g_{4}(K) \equiv \frac{2}{\pi} \mathcal{K}(r) \mathcal{K}^{\prime}(r)+\log r^{\prime} \\
&-\left(\frac{2}{\pi}\right)^{2} \frac{\mathcal{K}^{\prime}(r)}{\mathcal{K}(r)} \mathcal{K}(s)^{3}\left[\mathcal{E}^{\prime}(s)-s^{2} \mathcal{K}^{\prime}(s)\right] .
\end{aligned}\right.
$$


From [7, Theorem $3.21(1),(7)]$, we see that $g_{4}$ is strictly increasing on $(1, \infty)$. By $(1.23)$ and (1.19),

$$
\left\{\begin{aligned}
g_{4}(1)= & m\left(r^{\prime}\right)+\log r^{\prime}+m(r)-\frac{4}{\pi^{2}} \mathcal{K}(r)^{2} \mathcal{K}^{\prime}(r)\left[\mathcal{E}^{\prime}(r)-r^{2} \mathcal{K}^{\prime}(r)\right] \\
= & m\left(r^{\prime}\right)+\log r^{\prime}+\frac{4}{\pi^{2}} \mathcal{K}(r) \mathcal{K}^{\prime}(r)\left\{\frac{\pi}{2} r^{\prime 2}-\mathcal{K}(r)\left[\mathcal{E}^{\prime}(r)-r^{2} \mathcal{K}^{\prime}(r)\right]\right\} \\
= & m\left(r^{\prime}\right)+\log r^{\prime}+\frac{4}{\pi^{2}} \mathcal{K}(r) \mathcal{K}^{\prime}(r)\left\{r^{2} \mathcal{K}(r)\left[\mathcal{K}^{\prime}(r)-\mathcal{E}^{\prime}(r)\right]\right. \\
& \left.\quad-r^{\prime 2} \mathcal{K}^{\prime}(r)[\mathcal{K}(r)-\mathcal{E}(r)]\right\} \\
= & m\left(r^{\prime}\right)+\log r^{\prime}+m(r) m\left(r^{\prime}\right)\left\{\frac{\mathcal{K}^{\prime}(r)-\mathcal{E}^{\prime}(r)}{r^{\prime 2} \mathcal{K}^{\prime}(r)}-\frac{\mathcal{K}(r)-\mathcal{E}(r)}{r^{2} \mathcal{K}(r)}\right\} .
\end{aligned}\right.
$$

It follows from [28, Corollary $3.9(1)]$ and (4.33) that

$$
g_{4}(1)>0 \text { for } \quad r \in(0,1 / \sqrt{2}],
$$

and that

$$
g_{4}(1)>g_{5}(r) \equiv m\left(r^{\prime}\right)+\log r^{\prime}-\frac{1}{2} m(r) m\left(r^{\prime}\right)
$$

for $r \in[1 / \sqrt{2}, 1)$.

By [7, Theorem $3.30(1)]$ and Lemma 2.1 (1), we see that $g_{5}$ is strictly increasing on $[1 / \sqrt{2}, 1)$. Since

$$
g_{5}(1 / \sqrt{2})=\frac{1}{\pi} \mathcal{K}(1 / \sqrt{2})^{2}-\frac{1}{2 \pi^{2}} \mathcal{K}(1 / \sqrt{2})^{4}-\frac{1}{2} \log 2=0.148 \cdots>0,
$$

it follows from (4.35) that

$$
g_{4}(1)>0 \text { for } \quad r \in[1 / \sqrt{2}, 1) .
$$

It follows from (4.34), (4.36), (4.32), and the monotonicity of $g_{4}$ that $g_{3}$ is strictly increasing on $(1, \infty)$. Hence the monotonicity of $g$ follows from (4.31) and [7, Lemma 1.25].

The limiting values of $g$ follow from (4.31) and l'Hôpital's Rule. The second and third inequalities in (4.29) are clear, while the first follows from [32, Lemma 2.27]. Taking $t=1$ in (4.29), we get (4.30).

\section{Acknowledgments.}

This work was completed during the second author's visits to Michigan State University at East Lansing, MI, U.S.A., and to the University of Helsinki, Finland. His visits were supported by grants from the Department of Mathematics at Michigan State University, the Finnish Academy of Science and Letters, the Academy of Finland, and the Commission on Development and Exchanges in Paris. He wishes to express his thanks to these institutions. 


\section{References}

[1] S. Agard, "Distortion theorems for quasiconformal mappings," Ann. Acad. Sci. Fenn., Ser. AI 413 (1968), 1-12.

[2] S. Agard and F. W. Gehring, "Angles and quasiconformal mappings," Proc. London Math. Soc. 14 (1965), 1-21.

[3] G. D. Anderson, S.-L. Qiu, and M. K. Vamanamurthy, "Grötzsch ring and quasiconformal distortion functions," Hokkaido Math. J. 24 (1995), 551-566.

[4] _ "Inequalities for distortion functions and plane quasiconformal mappings," New Zealand J. Math. 26 (1997), 1-18.

[5] G. D. Anderson, S.-L. Qiu, and M. Vuorinen, "Bounds for the Hersch-Pfluger and Belinskii distortion functions," in Computational Methods and Function Theory 1997 (Nicosia) (N. Papamichael et al., eds.), Ser. Approx. Decompos., 11, World Sci. Publ., River Edge, NJ, 1999, pp. 9-22.

[6] G. D. Anderson and M. K. Vamanamurthy, "Some properties of quasiconformal distortion functions," New Zealand J. Math. 24 (1995), 1-15.

[7] G. D. Anderson, M. K. Vamanamurthy, and M. Vuorinen, Conformal Invariants, Inequalities, and Quasiconformal Maps, Wiley, New York, 1997.

[8] P. P. Belinskit, "General properties of quasiconformal mappings," Izdat. Nâuka, Sibirsk. Otdelenie, Novosibirsk, 1974 (Russian).

[9] B. C. Berndt, Ramanujan Notebooks, Part III, Springer-Verlag, New York, 1991.

[10] B. C. Berndt, S. Bhargava, and F. G. Garvan, "Ramanujan's theories of elliptic functions to alternative bases," Trans. Amer. Math. Soc. 347 (1995), 4163-4244.

[11] J. M. Borwein And P. B. Borwein, Pi and the AGM, Wiley, New York, 1987.

[12] F. Bowman, Introduction to Elliptic Functions with Applications, Dover, New York, 1961.

[13] P. F. Byrd and M. D. Friedman, Handbook of Elliptic Integrals for Engineers and Physicists, Grundlehren Math. Wiss., Vol. 57, Second ed., Springer-Verlag, Berlin-GöttingenHeidelberg, 1971.

[14] W. K. Hayman, "Some remarks on Schottky's theorem," Proc. Cambridge Philos. Soc. 43 (1947), 442-454.

[15] W. K. Hayman, Subharmonic Functions, Vol. 2, Academic Press, San Diego, 1989.

[16] C.-Q. He, Distortion estimates of quasiconformal mappings, Sci. Sinica Ser. A 27 (1984), $225-232$.

[17] J. Hempel, "Precise bounds in the theorems of Schottky and Picard," J. London Math. Soc. (2) 21 (1980), 279-286.

[18] J. Hersch and A. Pfluger, "Généralisation du lemme de Schwarz et du principe de la mesure harmonique pour les fonctions pseudo-analytiques," C. R. Acad. Sci. Paris 234 (1952), 43-45. 
[19] J. Jenkins, "On explicit bounds for Schottky's theorem," Canad. J. Math. 7 (1955), 76-82.

[20] O. Lehto and K. I. Virtanen, Quasiconformal Mappings in the Plane, 2nd ed., Die Grundlehren der math. Wiss., Band 126, Springer-Verlag, Berlin, Heidelberg, and New York, 1973.

[21] O. Lehto, K. I. Virtanen, and J. VÄis ÄLÄ, "Contributions to the distortion theory of quasiconformal mappings," Ann. Acad. Sci. Fenn. Ser. A I 273 (1959), 1-14.

[22] Z. Li And G. Cui, "A note on Mori's theorem of $K$-quasiconformal mappings," Acta Math. Sinica (N.S.) 9 (1993), 55-62.

[23] G. MARTin, "The distortion theorem for quasiconformal mappings, Schottky's theorem and holomorphic motions," Proc. Amer. Math. Soc. 125 (1997), 1095-1103.

[24] D. Partyka, "An alternative proof of a result due to Douady and Earle," Ann. Univ. Mariae Curie-Sktodowska 42 (1988), 58-68.

[25] D. PARTYKa And K.-I. SAKan, "On an asymptotically sharp version of Heinz's inequality," Ann. Acad. Sci. Fenn. Ser. A I Math. 30 (2005), 167-182.

[26] S.-L. QIU, "Distortion properties of $K$-q.c. maps and a better estimate of Mori's constant," Acta Math. Sinica 35 (4) (1992), 492-504.

[27] : "Agard's $\eta$-distortion function and Schottky's theorem," Sci. China Ser. A 26 (8) (1996), 683-690.

[28] S.-L. Qiu And M. K. Vamanamurthy, "Elliptic integrals and the modulus of Grötzsch ring," Panamer. Math. J. 5 (2) (1995), 41-60.

[29] S.-L. Qiu, M. K. Vamanamurthy, and M. Vuorinen, "Bounds for quasiconformal distortion functions," J. Math. Anal. Appl. 205 (1997), 43-64.

[30] "Some inequalities for the Hersch-Pfluger distortion function," J. Inequal. Appl. 4 (1999), 115-139.

[31] S.-L. QIU AND M. VuORInen, "Submultiplicative properties of the $\varphi_{K}$-distortion function," Studia Math. 117 (1996), 225-242.

[32] (Quasimultiplicative properties for the $\eta$-distortion function," Complex Variables Theory Appl. 30 (1996), 77-96.

[33] P. Seittenranta, "Möbius-invariant metrics," Math. Proc. Cambridge Philos. Soc. 125 (1999), 511-533.

[34] P. Tukia And J. VÄIsÄLÄ, "Quasisymmetric embeddings of metric spaces," Ann. Acad. Sci. Fenn., Ser. AI Math. 5 (1980), 97-114.

[35] J. VÄIsÄLё, “Quasimöbius maps,” J. Anal. Math. 44 (1984/85), 218-234.

[36] J. VÄIsÄLÄ, M. Vuorinen, And H. Wallin, "Thick sets and quasisymmetric maps," Nagoya Math. J. 135 (1994), 121-148.

[37] M. K. Vamanamurthy and M. Vuorinen, "Functional inequalities, Jacobi products and quasiconformal maps," Illinois J. Math. 38 (1994), 394-419. 
[38] M. Vuorinen, Conformal Geometry and Quasiregular Mappings, Lecture Notes in Math., Vol. 1319, Springer-Verlag, Berlin, Heidelberg, and New York, 1988.

[39] _ "Singular values, Ramanujan modular equations, and Landen transformation," Studia Math. 121 (1996), 221-230.

[40] E. T. Whittaker and G. N. Watson, A Course of Modern Analysis, 4th ed., Cambridge University Press, London and New York, 1958.

[41] S. Yamashita, "Inverse functions of Grötzsch's and Teichmüller's modulus functions," J. Math. Kyoto Univ. 43 (2004), 771-805.

[42] J. ZajA̧C, "The distortion function $\Phi_{K}$ and quasihomographies," in Current Topics in Analytic Function Theory (H. M. Srivastava et al., eds.), World Scientific Publ., 1992, pp. 403-428.

[43] S. Zhang, "On explicit bounds in Schottky's theorem," Complex Variables Theory Appl. 14 (1990), 15-23.

\author{
ANDERSON: \\ Department of Mathematics \\ Michigan State University \\ East Lansing, MI 48824 \\ U. S. A. \\ e-mail: anderson@math.msu.edu

\section{QIU:} \\ President \\ Zhejiang Sci-Tech University \\ Xiasha Higher Education Zone \\ Hangzhou 310038 \\ P. R. CHINA \\ VUORINEN: \\ Department of Mathematics \\ FIN-20014 University of Turku \\ FINLAND \\ e-mail: vuorinen@utu.fi
}

